The University of Maine

\title{
DigitalCommons@UMaine
}

Marine Sciences Faculty Scholarship

School of Marine Sciences

4-1-2000

\section{Animal Guts as Nonideal Chemical Reactors: Partial Mixing and Axial Variation in Absorption Kinetics}

Peter Jumars

University of Maine - Main, jumars@maine.edu

Follow this and additional works at: https://digitalcommons.library.umaine.edu/sms_facpub

\section{Repository Citation}

Jumars, Peter, "Animal Guts as Nonideal Chemical Reactors: Partial Mixing and Axial Variation in Absorption Kinetics" (2000). Marine Sciences Faculty Scholarship. 14.

https://digitalcommons.library.umaine.edu/sms_facpub/14 


\section{Animal Guts as Nonideal Chemical Reactors: Partial Mixing and Axial Variation in Absorption Kinetics}

Peter A. Jumars

Darling Marine Center, University of Maine, Walpole, Maine 04573-3307

Submitted November 30, 1998; Accepted November 22, 1999

ABSTRACT: Animal guts have been idealized as axially uniform plugflow reactors (PFRs) without significant axial mixing or as combinations in series of such PFRs with other reactor types. To relax these often unrealistic assumptions and to provide a means for relaxing others, I approximated an animal gut as a series of $n$ continuously stirred tank reactors (CSTRs) and examined its performance as a function of $n$. For the digestion problem of hydrolysis and absorption in series, I suggest as a first approximation that a tubular gut of length $L$ and diameter $D$ comprises $n=L / D$ tanks in series. For $n \geq 10$, there is little difference between performance of the $n$ CSTR model and an ideal PFR in the coupled tasks of hydrolysis and absorption. Relatively thinner and longer guts, characteristic of animals feeding on poorer forage, prove more efficient in both conversion and absorption by restricting axial mixing. In the same total volume, they also give a higher rate of absorption. I then asked how a fixed number of absorptive sites should be distributed among the $n$ compartments. Absorption rate generally is maximized when absorbers are concentrated in the hindmost few compartments, but high food quality or suboptimal ingestion rates decrease the advantage of highly concentrated absorbers. This modeling approach connects gut function and structure at multiple scales and can be extended to include other nonideal reactor behaviors observed in real animals.

Keywords: digestion, hydrolysis, absorption, reactor theory.

Digestive performance of guts has been modeled by analogy with engineered chemical reactors, most frequently with plug-flow reactors (PFRs). In an ideal PFR, axial mixing is insignificant relative to the flow rate of material through the gut; indigestible items leave in the same order that they enter. Almost from the outset, however, shortcomings became apparent in this mixing idealization

\footnotetext{
*E-mail: jumars@maine.edu.
}

Am. Nat. 2000. Vol. 155, pp. 544-555. (c) 2000 by The University of Chicago. 0003-0147/2000/15504-0009\$03.00. All rights reserved.
(Penry 1989; Martínez del Rio et al. 1994; Levey and Martínez del Rio 1999), and results of explicit experiments appear to contradict some predicted responses of ingestion rates and absorption efficiencies to food quality under an optimization premise (e.g., Karasov and Cork 1996; Jumars and Martínez del Rio 1999). Because the ideal mixing descriptions are known to be flawed, however, it cannot be clear whether failure of predictions is caused by inaccuracy of the optimization premise in favor of some other "operating policy," such as homeostasis (Calow 1982), or, instead, to this inaccuracy in reactor description.

Adding further doubt to present reactor-theory predictions (e.g., Jumars 2000, in this issue) is their assumption of axial uniformity in the distribution of hydrolytic and absorptive capacity. There clearly is need for a robust modeling approach that can accommodate both partial axial mixing and differentiation of function along the gut and that can deal with other foreseeable deviations from ideal reactor performance. Here, I adapt an approach used by chemical engineers (Fogler 1992) to admit both partial axial mixing and axial differentiation of absorptive function. The model reveals interaction between ingestion rate and location of absorptive sites in determining absorption rate, and maximal absorption rate occurs when absorptive sites are concentrated near the rear of the gut's hydrolytic and absorptive sections. This modeling approach thus has potential to predict linkages between gut function (processing rates) and structure from the level of gross morphology to the level of histology. It can also accommodate further modifications, such as axially varying hydrolytic capacities, lumen-tissue water fluxes (distinct hindgut functions), and detour of some digesta into ceca.

\section{Methods}

Mechanics of digesta movement through a tubular gut appear incompatible in general with the idea of simple, unidirectional flow characteristic of a PFR. Most portions of most guts are tubular (roughly cylindrical and longer than wide), but analogy of guts with ideal, engineered PFRs is strained. Unlike high-velocity gas-phase reactors typi- 


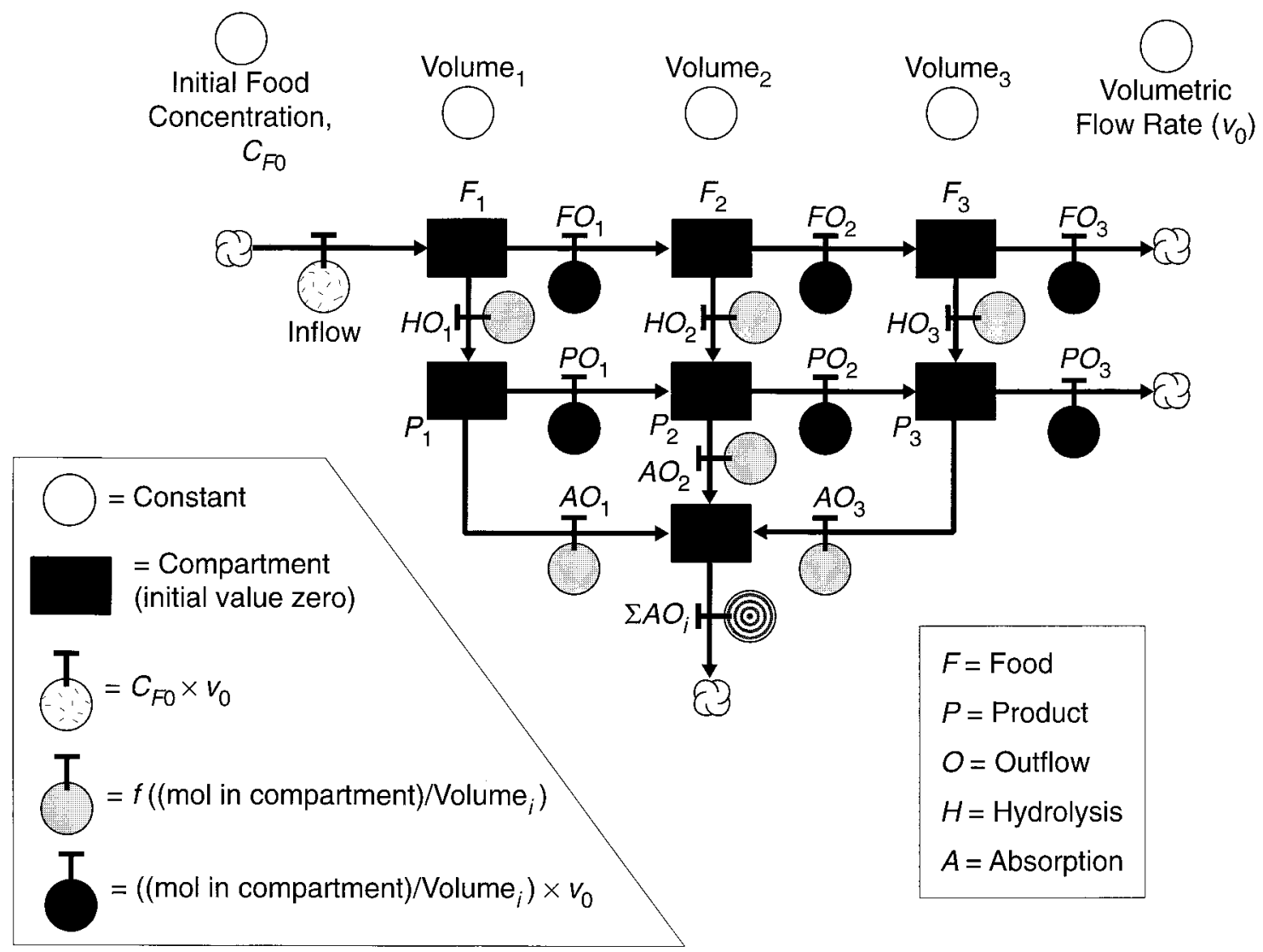

Figure 1: Diagram of the Stella compartment-model implementation for a gut comprising three continuously stirred tank reactors. For larger $n$, connections for end compartments remain the same, but the number of compartments sandwiched between them is varied. Total rate of absorption (flow through the control gate partially shaded with concentric circles) is determined by summing absorption from all product compartments after the model reaches steady state. For each initial food concentration and axial distribution of absorptive sites, volumetric flow rate is stepped until the volumetric flow rate that gives maximal absorption rate is found.

cally modeled as PFRs, flow of two-phase (solid-liquid) and highly viscous digesta is comparatively slow and never fully turbulent. Gut contents in most metazoans are both mixed and moved by muscular contractions (e.g., Macagno and Christensen 1981). It is impossible physically to have contraction of the circular muscles without causing fluid motion axially in both directions away from the point of contraction. Mixing induced by such contractions is constrained radially by the wall of the lumen. The cutoff is less abrupt axially, but mixing cells in fluids resist gross asymmetry, so that the characteristic axial length scale of mixing should approximate gut diameter or the (anti)peristaltic wavelength. Furthermore, wavelengths of (anti)peristaltic waves do not differ radically from gut diameter (e.g., Mathias and Sninsky 1985). These arguments make it logical to divide a tubular animal gut of length $L$ into a series of $n$ mixing cells, each roughly equal to the diameter $(D)$ of the gut lumen. Hence, $n$ is of order $L / D$.
Each mixing cell, in turn, is equated with a continuously stirred tank reactor (CSTR) in which mixing is instantaneous and complete in all directions.

I simulated coupled hydrolysis in and absorption from these linked CSTRs initially with Stella II and then checked them all with Stella 5 (High Performance Systems, Hanover, N.H.). Each calculation of the program is based on what is effectively a single CSTR, and Penry (1993) has pointed out the exact correspondence of a box or compartment in a "box" model with a CSTR. Conceptually, a PFR of volume $G$ is chopped into $n$ CSTRs connected in series, each of volume $G / n$. The number of boxes in a compartment-model simulation, however, is $n$ times the number of reactants being tracked (fig. 1). Here, there are only two reactants of interest, the food (at concentration $C_{\mathrm{F}}$ ) and its hydrolysate, or product (at concentration $C_{\mathrm{p}}$ ). I implemented mole balance explicitly; all state variables are in moles and all flows in moles per unit of time; I 
calculate concentrations from compartment volumes for intermediate kinetic calculations. The Stella implementation thus imposes the mass balances characteristic of CSTRs performing hydrolysis and absorption (eqq. [1], [3], and [4] of Jumars 2000, in this issue). I use equal volumes for the CSTRs for all the results reported here.

I explored linear, hyperbolic, and sigmoidal kinetics of digestion. Under linear kinetics, rates of hydrolysis in and absorption from the CSTR were proportional, respectively, to food and product concentrations in that compartment:

$$
\begin{aligned}
& -r_{\mathrm{FP}}=k C_{\mathrm{F}}, \\
& -r_{\mathrm{PA}}=a C_{\mathrm{P}},
\end{aligned}
$$

where subscripts $\mathrm{F}$ and $\mathrm{P}$ refer to food and product, respectively, and the double subscripts indicate rates $(r)$ of disappearance of food and product, respectively, by conversion of food to product (FP) and by conversion of product to absorbate (PA). For hyperbolic kinetics,

$$
\begin{aligned}
& -r_{\mathrm{FP}}=\frac{V_{\mathrm{max}} C_{\mathrm{F}}}{K_{m}+C_{\mathrm{F}}}, \\
& -r_{\mathrm{PA}}=\frac{W_{\max } C_{\mathrm{P}}}{M_{m}+C_{\mathrm{P}}},
\end{aligned}
$$

where $V_{\max }$ and $W_{\max }$ are the maximal rates of hydrolysis and absorption, respectively, and $K_{m}$ and $M_{m}$ are the respective half-saturation constants. For sigmoidal kinetics, I set

$$
\begin{aligned}
& -r_{F P}=\frac{V_{\max } C_{\mathrm{F}}^{2}}{K_{m}^{2}+C_{\mathrm{F}}^{2}}, \\
& -r_{P A}=\frac{W_{\max } C_{P}^{2}}{M_{m}^{2}+C_{\mathrm{P}}^{2}} .
\end{aligned}
$$

To facilitate comparison with results from ideal reactor models (Dade et al. 1990; Jumars and Martínez del Rio 1999; Jumars 2000, in this issue), I kept the kinetics of digestion and absorption identical $\left(-r_{\mathrm{FP}}=-r_{\mathrm{PA}}\right)$, except with respect to redistribution of absorptive sites. I used standard reactor-theory convention of double subscripting with the first subscript denoting the component ( $\mathrm{F}$ for food and $\mathrm{P}$ for product) and the second denoting incoming material $(0$, for initial or time 0$)$ and material leaving the last compartment ( $\mathrm{f}$ for final). Thus incoming food has concentration $C_{\mathrm{F} 0}$ and outgoing undigested food has concentration $C_{\mathrm{Ff}}$. As noted by Jumars (2000, in this issue), hyperbolic kinetics should characterize animals that have active uptake systems and that feed on simple substrates (e.g., disaccharides), whereas sigmoidal kinetics should be more typical of animals whose growth rates are limited by acquisition of more complicated materials through many reactions going on in parallel (e.g., protein hydrolysis and absorption).

The $n$ "tanks-in-series" model is a well-worked engineering approximation to intermediate cases between a CSTR and PFR, so solutions against which to check simulation results are available for some cases. In particular, simple analytic expressions are available for distributions of conservative tracers (no reaction) and for results of linear kinetics (Fogler 1992, pp. 762-765). I assumed throughout that volume of digesta is conserved and, hence, that the same flow rate holds for all tanks in the series. The expected residence-time distribution of a conservative tracer, then, is given nondimensionally as $E(Q)$, where $Q=t / \tau, t$ is residence time of the tracer, and $\tau$, or throughput time, has the usual continuous-flow reactor-theory interpretation of $G / v_{0}$ (eq. [14-18] of Fogler 1992, p. 764):

$$
E(\boldsymbol{\Theta})=\frac{n(n \Theta)^{n-1}}{(n-1) !} e^{-n \Theta}
$$

In the simulations, I started the first tank in the series at a fixed concentration of tracer and monitored exit concentration from the last tank. When total reactor volume is kept fixed, this procedure injects an absolute amount of tracer that is inversely proportional to the number of reactors in series, so the results can be easily rescaled.

Other solved $n$ CSTR cases that allowed me to check my simulation results included simple linear kinetics (Fogler 1992) and hyperbolic kinetics of hydrolysis (Luyben and Tramper 1982). Unfortunately, there is no compact solution for coupled hyperbolic or sigmoidal equations of hydrolysis and absorption (Dade et al. 1990; Jumars 2000, in this issue).

I present results for only a small set of simple choices for parameter values because they prove sufficient to demonstrate the basic behavior of the $n$ CSTR series an $n$ increases. Furthermore, published analyses for ideal reactors (e.g., Jumars 2000, in this issue) can already be used to calculate bounds on $n$ CSTR performance, with the single CSTR $(n=1)$ and the PFR $(n=\infty)$ at the two extremes. For the initial simulations, I set $G, C_{\mathrm{F} 0}, K_{m}, M_{m}, V_{\max }$, and $W_{\max }$ equal to 1 . The plots will apply directly, however, to data scaled suitably, as pointed out in the figure captions. One can also ask how performance should change with food quality. For direct comparison with ideal reactor behavior (Jumars 2000, in this issue) and to avoid speciesspecific details of processing costs, I treated neither variable enzyme secretion (time-varying $V_{\max }$ ) nor up- or down- 
regulation of absorbers (time-varying $W_{\max }$ ). I kept hydrolysis and absorption balanced $\left(V_{\max }=W_{\max }\right.$ and $K_{m}=$ $M_{m}$ ) to keep the nonlinearity of the coupled hydrolysisabsorption problem intact but changed food quality to be 0.1 , or 10 , times $K_{m}$.

In order to examine the effects of poorer or richer foods in determining performance, I repeated the simulations for $C_{\mathrm{F} 0}=0.1$ and 10 , respectively, leaving the other parameter values constant. Numerical adequacy was checked by halving the time step until results no longer changed.

To determine the optimal axial distribution of absorbers, I took advantage of the fact that $W_{\max }$, the saturating rate of absorption in either hyperbolic or sigmoidal kinetics, should be proportional to the number of absorptive sites (as can be seen from the derivation of Michaelis-Menten kinetics reproduced by Penry and Jumars [1987]). I kept gut-averaged $W_{\max }$ constant (and equal to $V_{\max }$ ) but allowed absorptive sites to be distributed uniformly among any number $(\leq n)$ of the gut compartments. For example, I examined performance of guts with all the absorptive sites in one compartment-effectively giving that compartment $n W_{\max }$ for its saturating absorption rate but leaving $M_{m}$ unchanged-and distributed across half of the compartments (which gave each absorptive compartment $2 W_{\max }$ as its saturation value). I also experimented with allowing those gut compartments that contained absorptive sites to have different numbers from one another, so long as gut-average $W_{\max }$ was maintained, but the effect was so small in comparison to varying the number of absorbing compartments that it made little difference, and I do not offer those results here.

Linear kinetics of absorption are generally associated with passive uptake. My approach with them was analogous to that with saturating kinetics but requires different interpretation with respect to gut physiology. Variation among compartments in $a$ is variation in permeability; those compartments without absorption are impermeable, whereas the others become proportionally more permeable. That is, I multiplied $a$ by the same factor that I used for $W_{\max }$, to allow the effects of regional specialization to be compared between linear and saturating kinetics. Within-compartment variations in the areal distribution of absorptive or permeable sites cannot be accommodated in the $n$ CSTR model because the assumption of perfect mixing makes all absorptive sites within a compartment equally accessible to all product molecules.

Any cost that increases with ingestion rate will slow ingestion rate below the one at which cost-free gross absorption rate is maximized. Shortage of food and predation hazard are two other potential drivers of slower throughput rates, so I also asked how reactor performance would decline as ingestion rates slowed below their optima.

\section{Results}

In simulations of passage of inert tracer the only radically different curve from all the others is for the single CSTR; the rest differ largely in skewness and variance of the residence time, which decrease toward 0 as PFR behavior is approached. Simulations (fig. 2) match equation (7).

When absorptive sites are distributed uniformly among all $n$ CSTRs and food concentration is at the half-saturation values for hydrolysis and absorption (food is of intermediate quality), maximal absorption rate shows only modest variation with $n$ (fig. 3). This result is anticipated from the modest difference between the CSTR and PFR in total absorption rate $(20 \%$ in the case of linear reactions and less under saturating kinetics; cf. Jumars 2000, in this issue). Optimal flow (ingestion) rates and efficiencies of absorption show greater sensitivity, as expected from behavior of the two end members (the CSTR and the PFR); relatively little benefit is achieved by increasing the number of CSTRs beyond 10 or 20 .

With the same number of absorptive sites distributed among a subset of the $n$ CSTRs, however, somewhat greater
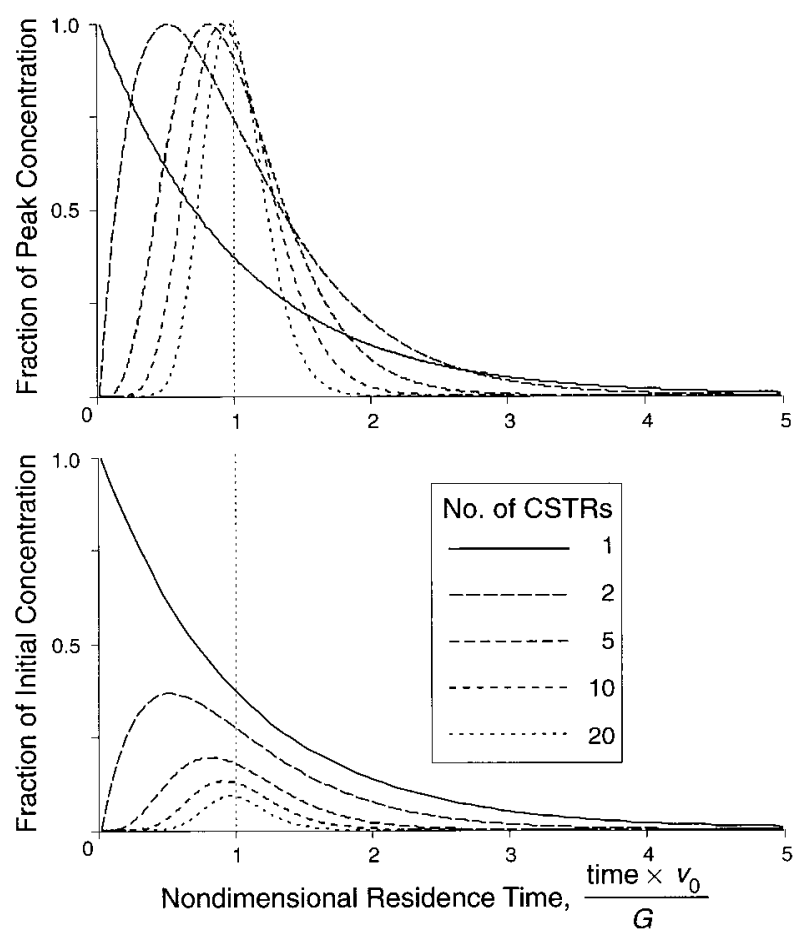

Figure 2: Hypothetical tracer results derived from the $n$ continuously stirred tank reactor model by starting the first compartment at a fixed tracer concentration at time 0 and monitoring the output stream continuously. Top panel, to allow shape comparisons of the curves, results are scaled to the maximal concentration observed in the output stream for given $n$. Bottom panel, results are scaled to the initial tracer concentration in the first compartment. 


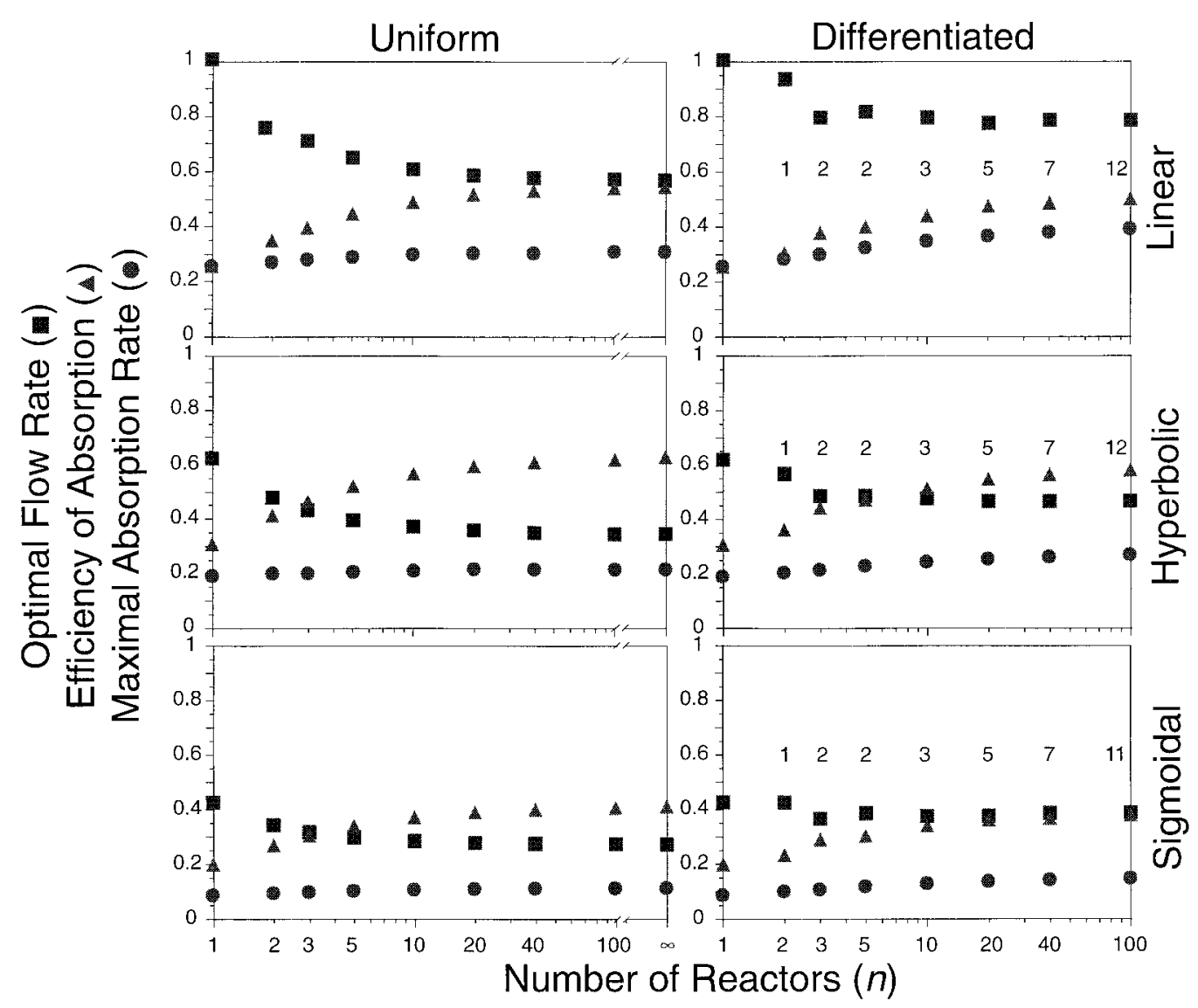

Figure 3: Optimal performance of $n$ continuously stirred tank reactor ( $n$ CSTR) series on food of intermediate quality as a function of $n$ for each of the three kinds of kinetics and for the cases where all CSTRs are absorbing (Uniform) or only the optimal number (printed in horizontal alignment to the symbols to which they refer) of rearmost compartments contain all the absorptive sites (Differentiated). Points plotted for $n=\infty$ are calculated from the plug-flow reactor performance equation (Jumars 2000); $n=1$ corresponds with a simple CSTR. For simplicity and ease of nondimensionalization, gut volume ( $G$, the sum over all compartments), $C_{\mathrm{F} 0}, K_{m}, M_{m}, V_{\max }$, and $W_{\max }$ were held at unity. Volumetric flow rate is $v_{0}$; efficiency of absorption is calculated as molar rate of absorption divided by the rate of ingestion; and maximal absorption rate $\left(\mathrm{mol}^{\mathrm{m}}\right.$ time $\left.\mathrm{e}^{-1}\right)$ is scaled by dividing it by the molar ingestion rate, $C_{\mathrm{F} 0} v_{0}$. This same plot would apply to both scaled flow rates $\left(v_{0} / G\right)$ at any gut volume and nondimensionalized concentrations (i.e., $C_{\mathrm{F} 0} / K_{m}=1$ ).

maximal absorption rates can be achieved (fig. 3 ) but at the expenses of lowered efficiency of absorption and increased rate of ingestion. Rather than staying nearly constant as $n$ increases, when absorbers are concentrated near the posterior of the gut, maximal absorption rate rises more steadily with $n$. Optimal ingestion rate, however, stays both higher and more constant with $n$ than when absorbers are distributed uniformly. With incoming food at the half-saturation concentration (fig. 3), saturation is evident for both hyperbolic and sigmoidal kinetics as a slowing of ingestion and absorption rates from the linear case. With uniform distribution of absorptive or permeable sites, however, all three kinds of kinetics show roughly twofold increase in absorption efficiency (defined as $1-$ $\left.\left[\left(C_{\mathrm{Ff}}+C_{\mathrm{Pf}}\right) / C_{\mathrm{F} 0}\right]\right)$ as $n$ goes from 1 to infinity. As expected from their increasingly complex kinetic constraints and the effects of partial saturation, absolute rates of gain fall from the linear to the hyperbolic to the sigmoidal case. There is only minor variation among the three kinds of kinetics, however, in optimal number of hindmost CSTRs containing absorptive or permeable walls.

When food concentration $\left(C_{\mathrm{F} 0}\right)$ is reduced to 0.1 times the half-saturation value for hydrolysis, maximal absorption rate for linear kinetics is simply reduced proportionately with the reduction in food concentration. Efficiency of absorption and optimal flow rate for linear kinetics do not change from figure 3 , so the linear case is not redrawn. Saturation effects are small under both hyperbolic and sigmoidal kinetics, and performance under hyperbolic kinetics (fig. 4) strongly resembles per- 


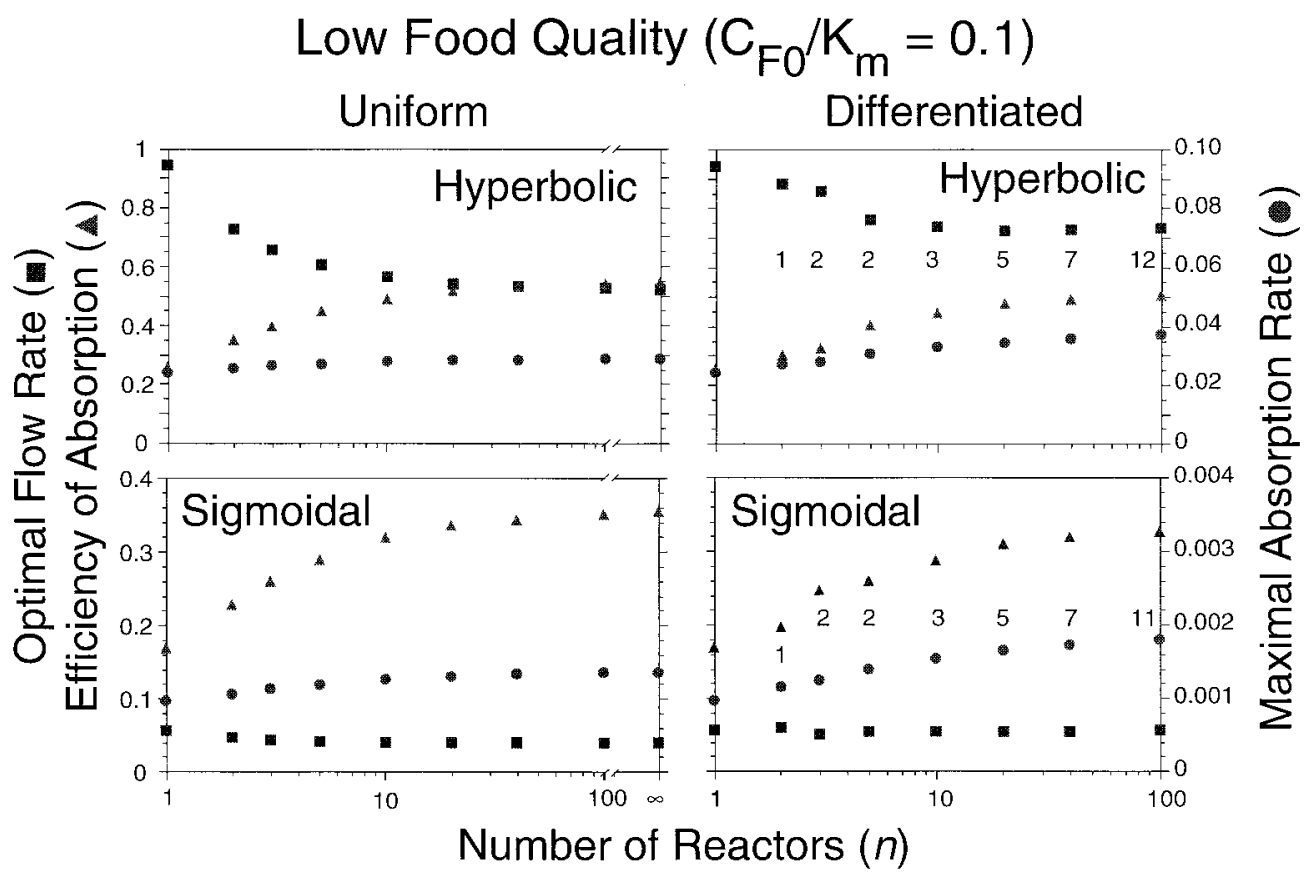

Figure 4: Optimal performance of $n$ continuously stirred tank reactor ( $n$ CSTR) series on food of low quality as a function of $n$ for hyperbolic kinetics and for the cases where all CSTRs are absorbing (Uniform) or only the optimal number (printed in horizontal alignment to the symbols to which they refer) of rearmost compartments contain all the absorptive sites (Differentiated). Points plotted for $n=\infty$ are calculated from the plug-flow reactor performance equation (Jumars 2000); $n=1$ corresponds with a simple CSTR. For simplicity and ease of nondimensionalization, gut volume ( $G$, the sum over all compartments), $K_{m}, M_{m}, V_{\max }$, and $W_{\max }$ were held at unity, but $C_{\mathrm{F} 0}$ was set at 0.1 . Volumetric flow rate is $v_{0}$; efficiency of absorption is calculated as molar rate of absorption divided by rate of ingestion; and maximal absorption rate $\left(\mathrm{mol}^{\mathrm{m}}\right.$ time $\left.\mathrm{e}^{-1}\right)$ is divided by the molar ingestion rate, $C_{\mathrm{F} 0} v_{0}$. This same plot would apply both to scaled flow rates $\left(v_{0} / G\right)$ at any gut volume as well as to nondimensionalized concentrations (i.e., $C_{\mathrm{Fo}} / M_{m}=0.1$ ).

formance under linear kinetics (because, for $C_{\mathrm{F} 0} \ll K_{m}$, $V$ approaches $C_{\mathrm{F} 0} V_{\max } / K_{m}$, i.e., linear behavior) but is not quite reduced to $10 \%$ of its value from $C_{\mathrm{F} 0}=1$ because of the saturation effects at the latter concentration. Rate of gain under sigmoidal kinetics (fig. 4) is reduced disproportionately further from the situation with $C_{\mathrm{F} 0}=1$ by two orders of magnitude instead of one. As anticipated from comparison of ideal CSTR and PFR performance (fig. 5B of Jumars 2000, in this issue), linear and hyperbolic (but not sigmoidal) kinetics show strong variation in optimal ingestion rate with varying $n$. Again, as for intermediate food quality, most of the variation is for $n<20$. Increasing $n$ in the uniform case again results in only mild increase of absorption rate, primarily through increased absorption efficiency. Increasing $n$ with axial differentiation in absorptive sites again yields higher rate of absorption but at the price of somewhat higher ingestion rates and lower absorption efficiencies than those for the uniform case. Interestingly, there is no change in optimal number of absorbing gut sections in going from intermediate to poor food quality.

As expected (cf. fig. 5 of Jumars 2000, in this issue), there is less difference with varying $n$ or between hyperbolic and sigmoidal kinetics when both are more nearly saturated at $C_{\mathrm{F} 0} / K_{m}=10$. Increasing $n$ again increases absorption efficiency in both uniform and differentiated guts under both hyperbolic and sigmoidal kinetics (fig. 5). Again, most of the change occurs for $n<20$. Optimal ingestion rates drop considerably from those on food of intermediate quality and yet remain high enough to show substantial saturation. Very little increase in maximal rate of absorption results as $n$ rises under uniform distribution of absorbers because optimal ingestion rate falls as absorption efficiency rises. A little more of the saturation is relieved by concentrating absorptive sites toward the posterior, allowing both absorption efficiency and absorption rate to increase with increasing $n$. At this higher food concentration and these slower ingestion rates, absorption rates are maximized when absorptive sites are spread among a larger number of the posterior compartments than was the case at lower food concentrations.

Performance of the $n$ CSTR model is similar to those of the CSTR and PFR when costs are considered and throughput is slowed below its cost-free optimum (fig. 6 herein 


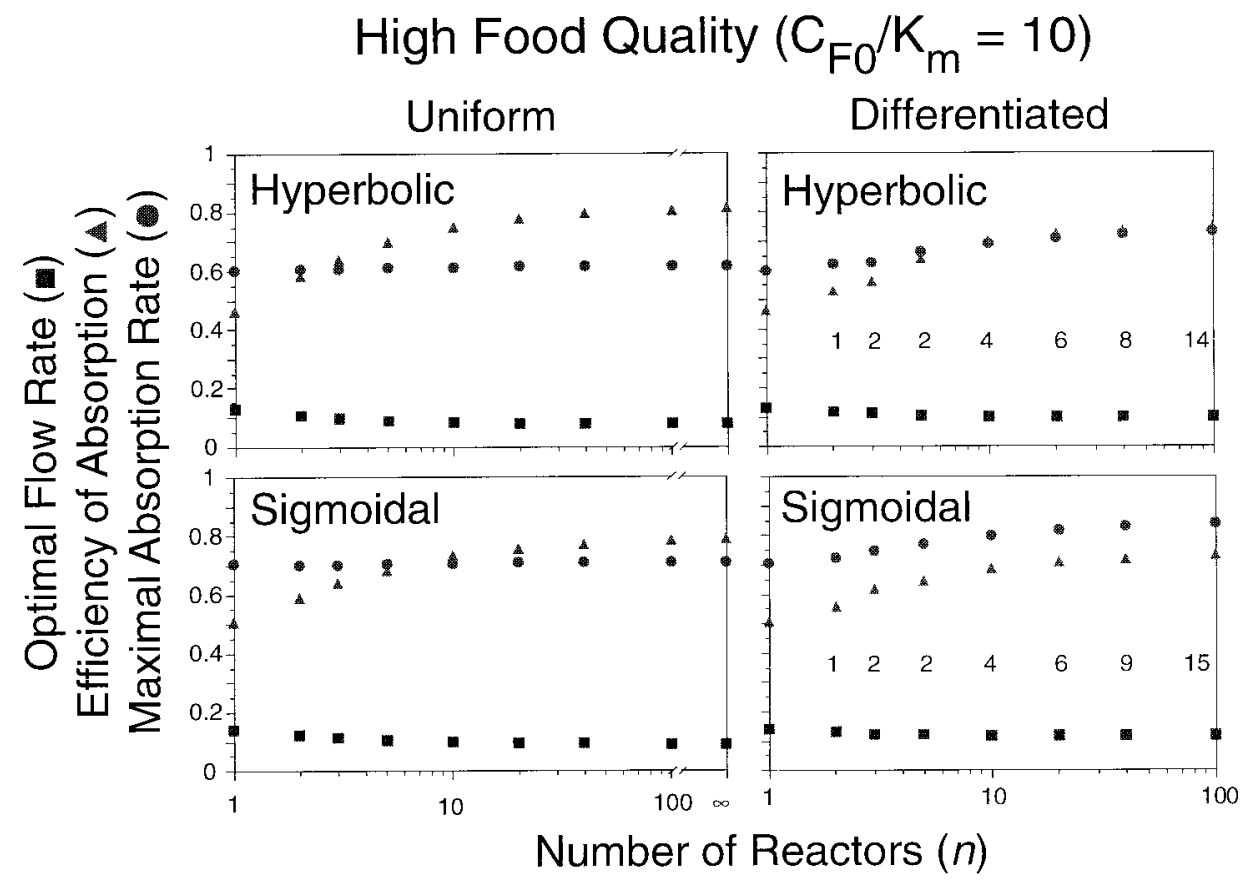

Figure 5: Optimal performance of $n$ continuously stirred tank reactor ( $n$ CSTR) series on food of high quality as a function of $n$ for hyperbolic kinetics and for the cases where all CSTRs are absorbing (Uniform) or only the optimal number (printed in horizontal alignment to the symbols to which they refer) of rearmost compartments contain all the absorptive sites (Differentiated). Points plotted for $n=\infty$ are calculated from the plug-flow reactor performance equation (Jumars 2000), and $n=1$ corresponds with a simple CSTR. For simplicity and ease of nondimensionalization, gut volume ( $G$, the sum over all compartments), $K_{\mathrm{m}}, M_{m}, V_{\max }$, and $W_{\max }$ were held at unity, but $C_{\mathrm{F} 0}$ was set at 10 . Volumetric flow rate is $v_{0}$; efficiency of absorption is calculated as molar rate of absorption divided by rate of ingestion; and maximal absorption rate $\left(\mathrm{mol}^{\mathrm{s}} \mathrm{t}^{-1}\right)$ is divided by the molar ingestion rate, $C_{\mathrm{F} 0} v_{0}$. This same plot would apply both to scaled flow rates $\left(v_{0} / G\right)$ at any gut volume as well as to nondimensionalized concentrations (i.e., $C_{\mathrm{F} 0} / M_{m}=10$ ).

vs. fig. 6 of Jumars 2000, in this issue), as it must be because the $n$ CSTR model is intermediate between the single CSTR and PFR ideals. Ingestion rate at which gross absorption rate is maximized and peakedness of the optimum, however, are sensitive to the axial distribution of absorptive sites. The more broadly distributed the absorptive sites, the slower the optimal ingestion rate and the higher the extraction efficiency. Conversely, concentration of absorptive sites in the posterior chambers allows product concentration to build to high levels before absorption begins, and high product concentration and high absorptive capacity combine to yield high absorption rates, but at the price of absorption efficiency.

Performance with $n / 2$ of the CSTRs having absorbers is not much degraded, however, from performance with the optimal number. Uniformly absorptive guts and guts with only the hindmost reactor being absorptive perform considerably more poorly and equally poorly with one another at some intermediate ingestion rate. At that point, summed absorption rates achieved in all the CSTRs at low product concentrations and absorber densities match the absorption rate achieved in one reactor at high product concentration and absorber density, giving the same absorption efficiency as well (fig. 6). Concentration of absorptive sites in only the hindmost chamber allows ample space and time for hydrolysis to proceed before absorption begins, but the reduction in absorption efficiency of product is severe when only one chamber is absorptive. Uniform distribution of absorbers has the opposite disadvantage: product concentrations are kept too low to drive absorption as rapidly as in the optimal distribution. An interesting aspect of reactor performance for all the cases simulated here is that the optimal distribution of absorptive sites retains its superiority at all suboptimal ingestion rates, so long as incoming food concentration and the kinetics of hydrolysis and absorption are not altered (for linear, hyperbolic, and sigmoidal kinetics). The more posteriorly concentrated the absorbers, the faster the ingestion rate at which absorption rate is maximized. Because qualitative behavior was similar for other food concentrations and numbers of reactors in series, only the results for $n=40$ are shown (fig. 6). 

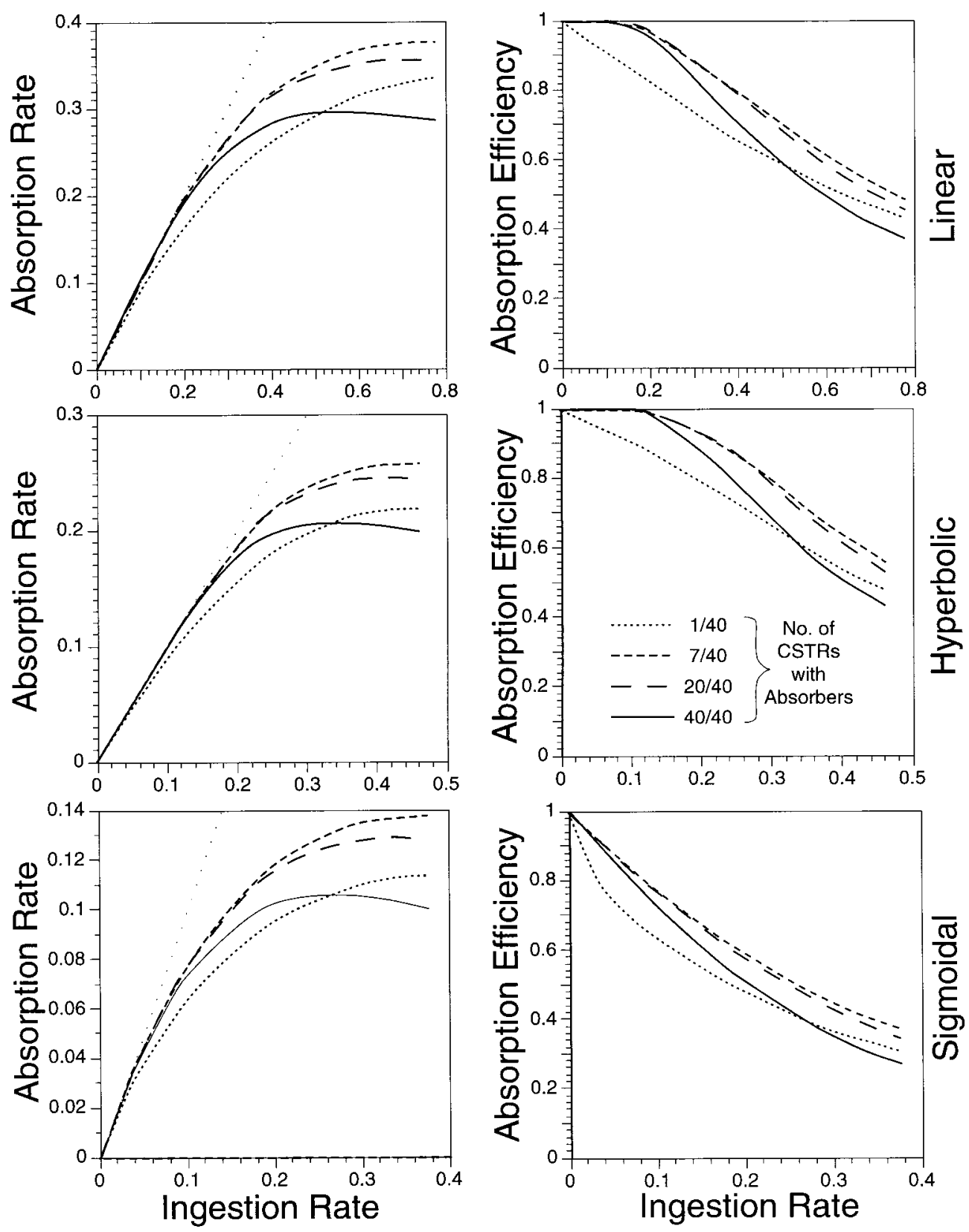

Figure 6: Performance of an $n$ continuously stirred tank reactor ( $n$ CSTR) reactor series for $n=40$ with ingestion rates at and below the optima that maximize absorption rate. $C_{\mathrm{F} 0}, K_{m}$, and $V_{\max }$, and $G$ were held at unity both for ease of nondimensionalization and for direct comparison with figure 3. Ingestion rate is scaled as $C_{\mathrm{F} 0} v_{0}$, and absorption rate is in the same units of moles per unit of time. Efficiency of absorption is calculated as molar rate of absorption divided by rate of ingestion. Absorption rate is expressed as a molar fraction of the ingestion rate, with the sparsely dotted straight line representing complete absorption. Note the scale changes on the axes. The best performance is achieved when all absorbers are placed in the hindmost 7/40 compartments; optimal ingestion rate varies inversely with the number of absorptive compartments. 


\section{Discussion}

Approximating the gut as a series of CSTRs has been suggested before in the context of guts (e.g., Penry and Jumars 1987; Hume 1989), based on the frequently used "tanksin-series" approximation in reactor engineering (Fogler 1992). Although a series of $n$ CSTRs rapidly approaches the behavior of a PFR of similar total volume as $n$ increases, the rate of approach depends on the specific kinetics involved (e.g., Luyben and Tramper 1982; Malcata 1988). This correspondence is often exploited to control reactions in chemical engineering applications when the medium is not inherently turbulent, that is, to make the reactor conform to the idealization rather than vice versa; the desirable characteristics of a PFR are obtained by connecting a set of well-mixed tanks in series, and it is tempting to speculate that evolution has found the same solution. Dellow et al. (1983) and Alexander (1993a) modeled segments of the guts of two different kinds of fermenters as series of four and three reactors, respectively. The novelty in my analysis is twofold. First is the suggestion that the model of a tubular gut as a series of $n$ CSTRs will be more realistic physically than one based on a PFR for any tubular gut section driven by circular muscle. Second is my systematic examination of coupled hydrolysis and absorption over a broad range of $n$, allowing me to assess how quickly the behavior of $n$ CSTRs in series approaches that of a single PFR as $n$ increases.

Outputs from the model (fig. 2) look remarkably like those from real-animal tracer experiments (e.g., Dellow 1982). Engineering practice is to estimate the effective number of reactors in series from the variances $\left(\sigma^{2}\right)$ in tracer output as (eq. [14-12] of Fogler 1992, p. 765)

$$
n=\frac{1}{\sigma_{\Theta}^{2}}=\frac{\tau^{2}}{\sigma_{t}^{2}} .
$$

It is usual in chemical engineering to accept noninteger values of $n$, in part because doing so allows one to calculate directly the chemical conversion achieved when reaction kinetics are linear (Fogler 1992). For nonlinear kinetics, however, there is no analogous general solution, and the consequent need to perform mole balances tank by tank makes a noninteger solution unacceptable. Equation (8) thus can be used both to test the rough approximation that $n$ should be near $L / D$ and to replace $n$ with a more accurate value based on tracer data. There is some suggestion from tracer output, however, that apparent $n$ may covary positively with ingestion rate (Levey and Martínez del Rio 1999). A likely mechanism is less variation in frequency of mixing contractions than in volumetric flow rate, leading to greater axial homogenization and, hence, smaller calculated and effective $n$ at slower throughput rates.

Caution is required, however, when comparing these tracer predictions against data. A simplification admitted by Penry and Jumars $(1986,1987)$ and further justified by Martínez del Rio and Karasov (1990) and Dade et al. (1990) is the assumption that equations for homogeneous catalysis (liquid phase only) apply to the heterogeneous catalysis of particulate foods. Both the PFR and CSTR equations assume that all enzyme and substrate molecules are in effective contact, which is part and parcel of the assumption of perfect and instantaneous radial mixing in both kinds of reactors. When food in the lumen occurs as large particles (whose internal portions are not in contact with hydrolytic enzymes) or when radial fluid mixing is incomplete, models that assume homogeneous catalysis will clearly overestimate instantaneous conversion and absorption rates because they will overestimate effective concentrations of reactants that are in contact for reaction. The assumption of homogeneous catalysis, however, does not require that the particles of food be well mixed radially, only that the fluid be brought into effective contact with both food and absorptive surfaces. Failure of particulate tracers to show perfect radial mixing —or even any mixing at all (cf. Penry 1989 for the holothuroid Parastichopus californicus) - therefore does not falsify this assumption. A conservative fluid tracer is needed for this critical test and, by this argument, should be used in preference to any particulate tracer if $n$ is to be estimated empirically for animals to test reaction-rate predictions based on homogeneous catalysis. A further complication in applying the empirical estimate of $n$ from a tracer is that the output of feces by most animals is discontinuous, making the empirical estimate of $n$ inherently less precise than in a typical engineering application.

As has been found with most other chemical kinetics, approach toward PFR performance for coupled hydrolysis and absorption is rapid with increasing numbers of CSTRs in series (figs. 3-5). What sets digestive applications apart from more usual engineering treatments, however, is the drawing-off of products by absorption through a substantial portion of the reactor rather than through collecting them only at the outlet. Although a great deal about the kinetics of digestion can be learned by studying engineering examples of reactions in series (e.g., Froment and Bischoff 1979 , p. 19), this spatially distributed absorption pervades the quantification of digestive kinetics and needs separate attention. Both here and in the engineering literature, however, the implication is that, with two reactions in series, the second cannot begin until there are some products from the first, but thereafter both may continue; in this case, some hydrolysis must precede ab- 
sorption, but further hydrolysis is not precluded when absorption begins.

These results indicate that PFR-based predictions (Dade et al. 1990; Martínez del Rio and Karasov 1990; Jumars 2000 , in this issue) should fit quite closely with results for guts that show some axial mixing but for which $n>10$. If the scaling argument $(n \approx L / D)$ used here proves adequate, PFR-based predictions should serve well for most tubular gut sections. A major benefit of reduced axial mixing from increasing $n$ is a reduction in the ingestion rate required to maximize absorption rate and the likely correlative reduction in risk of being taken by predators. As stressed elsewhere (Jumars 2000, in this issue), however, the rates and efficiencies predicted apply more appropriately to material exiting the midgut than to material leaving the gut as a whole: hindguts generally have some storage and recovery functions and show microbial activities that influence conversion efficiencies; they, however, are not modeled here.

Increase in absorption efficiencies and rates with increasing $n$ (irrespective of localization of absorptive sites) is one likely reason for the frequently observed increase in gut aspect ratios among closely related species as food quality decreases (e.g., Steiner 1994). I emphasize that the advantage noted here is dependent on the ratio of gut length to width $(n)$ in restricting axial mixing and not on absolute dimensions. A more obvious reason for absolutely small gut diameter is the benefit of accelerated diffusion: characteristic diffusion times scale roughly linearly with the square of the diffusion distance (Crank 1975). Both by restricting axial mixing and by speeding radial diffusional transport, smaller gut diameter can be expected to increase absorption efficiency. The latter effect is not modeled here but may be of great importance.

Maximal absorption rates occur when a small number of the hindmost CSTRs contain all the absorptive sites. That positioning is understandable because the reactions of interest are in series (hydrolysis followed by absorption), and higher product concentration drives absorption rate higher for all the kinetics considered here. If all the absorptive sites are located in the hindmost CSTR, however, efficiency of absorption is low enough to drive total absorption rate lower than if the same number of absorptive sites are distributed among a few posteriorly located reactors. Again, as in the previous discussion of conversion and absorption efficiencies, this prediction should translate into roughly uniform distribution of absorptive sites in a posterior portion of the invertebrate midgut or vertebrate small intestine. Hindguts and recta constitute sites for passive absorption of microbial by-products (and water in terrestrial animals) and for storage of feces before defecation. The ideal-reactor analog of foregut and midgut for effectively infinite $n$ would be two PFRs in series, with only hydrolysis occurring in the anterior one and both hydrolysis and absorption occurring in the posterior one. Inclusion of digestive costs, shortage of food, or hazard of predation may slow ingestion rates, however, decreasing the advantage of rearward concentration of absorbers.

I focused on variable axial mixing and localization of absorptive sites because they are the most frequent grounds for criticism of ideal reactor models and because they can be examined without species-specific details. Many other ideal-reactor assumptions can be relaxed through the $n$ CSTR approximation and may have even more important consequences for reactor performance. The $n$ CSTR model is preadapted to differential flow of fluids and solids (as is commonly observed; see, e.g., Stevens and Hume 1995) because product and food streams are distinguished (fig. 1). Moreover, water balance can be added to the constraints imposed by adding water as a separate flow stream for which mole balance also is maintained. If water is tracked explicitly in this fashion, then it becomes superior to gut dimensions for determining reactant concentration. Because water makes up substantial fractions of digesta volume in many species, it might also be treated efficiently as a primary determinant of compartmental volume in adaptive models wherein compartmental volume is allowed to vary with time and diet. Volume reduction during digestion can be substantial and can add appreciably to the rate of ingestion that a gut of a given volume can handle (e.g., Lehman 1976). A cecum or diverticulum is easy to incorporate as a side branch on figure 1, with bidirectional flow (in and out) from and to the lumen as well as absorption from and secretion into the invagination. Computer simulation has the added advantage of dealing straightforwardly both with time-varying ingestion rates, which are more the rule than the exception, and with time variation from up- or down-regulation of hydrolysis or absorption. Many of these additional departures from ideal reactor behavior, however, require tailoring to individual trophic groups or taxa.

Animal guts are diverse (Morton 1979) and no simple ideal model or single modification approach can fit them all. Whereas the $n$ CSTR model can deal with incomplete mixing in the axial dimension, it cannot do so radially. Chemical reactor theorists, therefore, have developed a slightly more complicated "dispersion model" that is capable of treating this case (Levenspiel 1972, pp. 272-290). Several related modifications for specific digestive flow patterns already have been made or suggested: refeed via coprophagy in lagomorphs and some rodents (Penry and Jumars 1987; Alexander 1993b), bypass and its implied dead space in deposit feeders (Penry 1989), and reflux and differential transport of lipids in seabirds (Place 1992). It is possible to treat these issues with $n$ CSTR variants, but chemical reactor engineers have developed simpler ap- 
proaches that may be superior for some applications (Fogler 1992). Also of potential interest in understanding guts is the recent development of membrane reactors and mod els of them (Govind and Itoh 1989): the analogy with peritrophic membranes (Peters 1992) and the potential for countercurrent flow (e.g., Treherne 1959) deserve to be explored. It is certain that more modifications of reactor theory will be needed both in applications to digestion and in future generalizations that include other organ systems and processes (e.g., water balance).

Neither variable axial mixing nor local concentration of absorptive sites now appears able to solve the problem of chronically low absorption efficiencies predicted by idealized reactor-theory models based on the optimization premise. The cause of this discrepancy appears to lie elsewhere, that is, with failure to account for postmidgut processes and the full suite of costs of feeding (Jumars 2000, in this issue), with failure to incorporate water balance among the constraints (Jumars and Martínez del Rio 1999), with other failures to include volume changes dur $\rightarrow$ ing digestion, or with failure of the optimization premise. On the path toward identifying and quantifying important constraints and operating characteristics of digestion ir real animals, pinpointing the causes is a high priority for the application of both physically and chemically credible nonideal reactor models.

\section{Acknowledgments}

This work was supported by National Science Foundation grant OCE 96-17701. I thank B. Krieger-Brockett for recommending Fogler (1992) as a general reference on nonideal reactors. R. Huey, C. Martínez del Rio, D. Penry, J. Schmidt, J. Travis, A. Woods, and an anonymous reviewer provided useful criticism of earlier drafts.

\section{Literature Cited}

$\rightarrow$ Alexander, R. M. 1993a. The energetics of coprophagy: a theoretical analysis. Journal of Zoology (London) 230: 629-637.

$\rightarrow-1993 \mathrm{~b}$. The relative merits of foregut and hindgut fermentation. Journal of Zoology (London) 231: 391-401.

$\rightarrow$ Calow, P. 1982. Homeostasis and fitness. American Nat uralist 120:416-419.

Crank, J. 1975. The mathematics of diffusion. Oxford University Press, Oxford.

Dade, W. B., P. A. Jumars, and D. L. Penry. 1990. Supply $\rightarrow$ side optimization: maximizing absorptive rates. Pages 531-556 in R. N. Hughes, ed. Behavioural mechanisms of food selection. Springer, Berlin.

$\rightarrow$ Dellow, D. W. 1982. Studies on the nutrition of macropodine marsupials. III. The flow of digesta through the stomach and intestine of macropodines and sheep. Australian Journal of Zoology 30:751-765.

Dellow, D. W., J. V. Nolan, and I. D. Hume. 1983. Studies on the nutrition of macropodine marsupials. V. Microbial fermentation in the forestomach of Thylogale thetis and Macropus eugenii. Australian Journal of Zoology 31: 433-443.

Fogler, H. S. 1992. Elements of chemical reaction engineering. 2d ed. Prentice Hall, Upper Saddle River, N.J.

Froment, G. F., and K. B. Bischoff. 1979. Chemical reactor analysis and design. Wiley, New York.

Govind, R., and N. Itoh, eds. 1989. Membrane reactor technology. American Institute of Chemical Engineering, New York.

Hume, I. D. 1989. Optimal digestive strategies in mammalian herbivores. Physiological Zoology 62:1145-1163.

Jumars, P. A. 2000. Animal guts as ideal chemical reactors: maximizing absorption rates. American Naturalist 155: 000-000.

Jumars, P. A., and C. Martínez del Rio. 1999. The tau of continuous feeding on simple foods. Physiological and Biochemical Zoology 72:633-641.

$\rightarrow$ Karasov, W. H., and S. J. Cork. 1996. Test of a reactorbased digestion optimization model for nectar-eating rainbow lorikeets. Physiological Zoology 69:117-138.

$\rightarrow$ Lehman, J. T. 1976. The filter-feeder as an optimal forager, and the predicted shapes of feeding curves. Limnology and Oceanography 21:501-516.

Levenspiel, O. 1972. Chemical reaction engineering. $2 \mathrm{~d}$ ed. Wiley, New York.

Levey, D. J., and C. Martínez del Rio. 1999. Test, rejection, and reformulation of a chemical reactor-based model of gut function in a fruit-eating bird. Physiological and Biochemical Zoology 72:369-383.

$\rightarrow$ Luyben, K. C. A. M., and J. Tramper. 1982. Optimal design for continuous stirred tank reactors in series using Michaelis-Menten kinetics. Biotechnology and Bioengineering 24:127-1220.

Macagno, E. O., and J. Christensen. 1981. Fluid mechanics of gastrointestinal flow. Pages 335-358 in L. R. Johnson, J. Christensen, M. I. Grossman, E. D. Jacobson, and S. G. Schultz, eds. Vol. 1. 2d ed. Physiology of the gastrointestinal tract. Raven, New York.

Malcata, F. X. 1988. Optimal design on an economic basis for continuous stirred tank reactors in series using Michaelis-Menten kinetics for ping-pong reactions. $\mathrm{Ca}-$ nadian Journal of Chemical Engineering 66:168-172.

Martínez del Rio, C., and W. H. Karasov. 1990. Digestion strategies in nectar- and fruit-eating birds and the sugar composition of plant rewards. American Naturalist 136: 618-637.

Martínez del Rio, C., S. J. Cork, and W. H. Karasov. 1994. Modelling gut function: an introduction. Pages 25-53 
in D. J. Chivers and P. Langer, eds. 1994. The digestive system in mammals: food, form and function. Cambridge University Press, Cambridge.

Mathias, J. R., and C. A. Sninsky. 1985. Motility of the small intestine: a look ahead. American Journal of Physiology 248:G495-G500.

Morton, J. 1979. Guts. 2d ed. University Park Press, Baltimore.

$\rightarrow$ Penry, D. L. 1989. Tests of kinematic models for depositfeeder guts: patterns of sediment processing by Parastichopus californicus (Stimpson) (Holothuroidea) and Amphicteis scaphobranchiata Moore (Polychaeta). Journal of Experimental Marine Biology and Ecology 128: 127-146.

1993. Digestive constraints on diet selection. Pages 32-55 in R. N. Hughes, ed. Diet selection. Blackwell Scientific, Oxford.

$\rightarrow$ Penry, D. L., and P. A. Jumars. 1986. Chemical reactor analysis and optimal digestion theory. BioScience 36: 310-315.
1987. Modeling animal guts as chemical reactors. American Naturalist 129:69-96.

Peters, W. 1992. Peritrophic membranes. Springer, Berlin. Place, A. R. 1992. Bile is essential for lipid assimilation in Leach's storm petrel Oceanodroma leucorhoa. American Journal of Physiology 263:R389-R399.

Steiner, G. 1994. Variations in the number of intestinal loops in Scaphopoda (Mollusca). Publications of the Statzione Zoologica Napoli Italy in Marine Ecology 15: $165-174$.

Stevens, C. E., and I. D. Hume. 1995. Comparative physiology of the vertebrate digestive system. $2 \mathrm{~d}$ ed. Cambridge University Press, Cambridge.

Treherne, J. E. 1959. Amino acid absorption in the locust (Schistocerca gregaria Forsk.) Journal of Experimental Biology 36:533-545.
Editor: Joseph Travis Associate Editor: Raymond B. Huey 